\title{
A new kind of uniqueness theorems for inverse Sturm-Liouville problems
}

Yuri Ashrafyan*

"Correspondence:
yuriashrafyan@ysu.am
Department of Mathematics and
Mechanics, Yerevan State University,
Alex Manoogian 1, Yerevan, 0025,
Armenia

\section{Abstract}

We prove Marchenko-type uniqueness theorems for inverse Sturm-Liouville problems. Moreover, we prove a generalization of Ambarzumyan's theorem.

Keywords: inverse problem; Sturm-Liouville operator; uniqueness theorem; Ambarzumyan theorem

\section{Introduction}

Let us denote by $L(q, \alpha, \beta)$ the Sturm-Liouville boundary value problem

$$
\begin{aligned}
& -y^{\prime \prime}+q(x) y=\mu y, \quad x \in(0, \pi), \mu \in \mathbb{C}, \\
& y(0) \cot \alpha+y^{\prime}(0)=0, \quad \alpha \in(0, \pi), \\
& y(\pi) \cot \beta+y^{\prime}(\pi)=0, \quad \beta \in(0, \pi),
\end{aligned}
$$

where $q$ is a real-valued, summable function, $q \in L_{\mathbb{R}}^{1}(0, \pi)$. At the same time, $L(q, \alpha, \beta)$ denotes the self-adjoint operator generated by problem (1.1)-(1.3) (see, e.g., [1-3]). It is known that under the above conditions the spectrum of operator $L(q, \alpha, \beta)$ is discrete and consists of real, simple eigenvalues (see, e.g., [2, 4]), which we denote by $\mu_{n}=\mu_{n}(q, \alpha, \beta)$, $n \geq 0$, emphasizing the dependence of $\mu_{n}$ on $q, \alpha$ and $\beta$. We assume that eigenvalues are enumerated in the increasing order, i.e.,

$$
\mu_{0}(q, \alpha, \beta)<\mu_{1}(q, \alpha, \beta)<\cdots<\mu_{n}(q, \alpha, \beta)<\cdots .
$$

Let $\varphi(x, \mu)$ be a solution of equation (1.1), which satisfies the initial conditions

$$
\varphi(0, \mu)=1, \quad \varphi^{\prime}(0, \mu)=-\cot \alpha
$$

The eigenvalues $\mu_{n}=\mu_{n}(q, \alpha, \beta), n \geq 0$, of $L(q, \alpha, \beta)$ are the solutions of equation

$$
\varphi(\pi, \mu) \cot \beta+\varphi^{\prime}(\pi, \mu)=0 .
$$

\section{Springer}

(c) The Author(s) 2017. This article is distributed under the terms of the Creative Commons Attribution 4.0 International License (http://creativecommons.org/licenses/by/4.0/), which permits unrestricted use, distribution, and reproduction in any medium, provided you give appropriate credit to the original author(s) and the source, provide a link to the Creative Commons license, and indicate if changes were made. 
It is easy to see that the functions $\varphi\left(x, \mu_{n}\right), n \geq 0$, are the eigenfunctions corresponding to the eigenvalue $\mu_{n}$. The squares of the $L^{2}$-norm of these eigenfunctions

$$
a_{n}=a_{n}(q, \alpha, \beta):=\int_{0}^{\pi}\left|\varphi\left(x, \mu_{n}\right)\right|^{2} d x, \quad n \geq 0,
$$

are called norming constants. The eigenvalues and norming constants are called spectral data (besides these, there are other quantities, which are also called spectral data). The inverse Sturm-Liouville problem is to reconstruct the quantities $q, \alpha, \beta$ by some spectral data.

Let $L=L(q, \alpha, \beta)$ and $L_{0}=L\left(q_{0}, \alpha_{0}, \beta_{0}\right)$ be two operators. The following assertion is usually called the uniqueness theorem of Marchenko. ${ }^{\mathrm{a}}$

Theorem 1.1 (Marchenko [5]) Let $q \in L_{\mathbb{R}}^{1}(0, \pi)$. If

$$
\begin{aligned}
& \mu_{n}(q, \alpha, \beta)=\mu_{n}\left(q_{0}, \alpha_{0}, \beta_{0}\right), \\
& a_{n}(q, \alpha, \beta)=a_{n}\left(q_{0}, \alpha_{0}, \beta_{0}\right),
\end{aligned}
$$

for all $n \geq 0$, then $\alpha=\alpha_{0}, \beta=\beta_{0}$ and $q(x)=q_{0}(x)$ almost everywhere.

One of the results of the present paper is the following theorem which, in some sense, is a generalization of Marchenko's uniqueness theorem.

Theorem 1.2 Let $q^{\prime} \in L_{\mathbb{R}}^{2}(0, \pi)$. If

$$
\begin{aligned}
& \mu_{n}\left(q, \alpha_{0}, \beta\right)=\mu_{n}\left(q_{0}, \alpha_{0}, \beta_{0}\right), \\
& a_{n}\left(q, \alpha_{0}, \beta\right) \geq a_{n}\left(q_{0}, \alpha_{0}, \beta_{0}\right),
\end{aligned}
$$

for all $n \geq 0$, then $\beta=\beta_{0}$ and $q(x) \equiv q_{0}(x)$.

This kind of uniqueness theorem has not been considered before. The main difference between Theorems 1.1 and 1.2 is that we replace the equality in (1.6) with the inequality in (1.8). Note that we assume $q^{\prime} \in L_{\mathbb{R}}^{2}(0, \pi)$ instead of general $q \in L_{\mathbb{R}}^{1}(0, \pi)$ since our proof is based on the results of Jodeit and Levitan (see [6]). And the parameter $\alpha$ of boundary condition is in advance fixed $\alpha=\alpha_{0}$.

Remark 1 Some analogues of Theorem 1.2 will be stated in the Appendix.

Historically, the first work in the theory of inverse spectral problems for Sturm-Liouville operators belongs to Ambarzumyan [7]. He proved that if the eigenvalues of SturmLiouville operator with Neumann boundary conditions are $n^{2}$, then the potential $q$ is 0 on $[0, \pi]$. It is known that the eigenvalues $\mu_{n}(0, \pi / 2, \pi / 2)$ of operator $L(0, \pi / 2, \pi / 2)$ are $n^{2}, n \geq 0$. The classical Ambarzumyan theorem in our notations will be as follows.

Theorem 1.3 (Ambarzumyan [7]) If $\mu_{n}(q, \pi / 2, \pi / 2)=\mu_{n}(0, \pi / 2, \pi / 2)=n^{2}$ for all $n \geq 0$, then $q(x) \equiv 0$. 
This was an exception as in general additional information was needed in order to reconstruct the potential $q$ uniquely. There are many generalizations of Ambarzumyan's theorem in various directions, we mention several of them (see, e.g., [8-15] and the references therein).

Our generalization of Ambarzumyan's theorem is as follows.

Theorem 1.4 Let $q^{\prime} \in L_{\mathbb{R}}^{2}(0, \pi)$.

$$
\text { If } \mu_{n}(q, \alpha, \pi-\alpha)=\mu_{n}(0, \alpha, \pi-\alpha) \text { for all } n \geq 0 \text {, then } q(x) \equiv 0 \text {. }
$$

We think that Theorem 1.4 is a natural generalization, because we use only one spectrum to reconstruct the potential $q$ without any additional conditions, as it is in the classical result.

\section{Preliminaries}

Two operators $L=L(q, \alpha, \beta)$ and $L_{0}=L\left(q_{0}, \alpha_{0}, \beta_{0}\right)$ are called isospectral if they have the same spectra, i.e., $\mu_{n}(q, \alpha, \beta)=\mu_{n}\left(q_{0}, \alpha_{0}, \beta_{0}\right), n \geq 0$. In what follows, if a certain symbol $\gamma$ denotes an object related to $L$, then $\gamma_{0}$ (or $\gamma^{0}$ depending on situation) will denote a similar object related to $L_{0}$.

The problem of describing all the operators $L$ isospectral with $L_{0}$ first was considered by Trubowitz et al. (see [16-19]) for $q \in L_{\mathbb{R}}^{2}(0, \pi)$. The same problem was considered by Jodeit and Levitan in [6] for $q$ such that $q^{\prime} \in L_{\mathbb{R}}^{2}(0, \pi)$. For this aim the Gelfand-Levitan integral equation and transformation operators were used in [6]. They constructed the kernel $F(x, y)$ of the integral equation as follows. Let $c_{n}, n \geq 0$, be arbitrary real numbers converging to zero, as $n \rightarrow \infty$, so rapidly that the function

$$
F(x, y)=\sum_{n=0}^{\infty} c_{n} \varphi_{0}\left(x, \mu_{n}^{0}\right) \varphi_{0}\left(y, \mu_{n}^{0}\right)
$$

is continuous and all the second order partial derivatives are also continuous. The integral equation

$$
K(x, y)+F(x, y)+\int_{0}^{x} K(x, t) F(t, y) d t=0, \quad 0 \leq y \leq x \leq \pi,
$$

is called Gelfand-Levitan integral equation. ${ }^{\mathrm{b}}$

They proved that if $1+c_{n} a_{n}^{0}>0$ for all $n \geq 0$, then the integral equation (2.2) has a unique solution $K(x, y)$ and the function

$$
\varphi(x, \mu)=\varphi_{0}(x, \mu)+\int_{0}^{x} K(x, t) \varphi_{0}(t, \mu) d t
$$

is a solution of the differential equation (1.1), with potential function

$$
q(x)=q_{0}(x)+2 \frac{d}{d x} K(x, x)
$$

and $\varphi(x, \mu)$ satisfies the initial conditions

$$
\varphi(0, \mu)=1, \quad \varphi^{\prime}(0, \mu)=-\cot \alpha,
$$


where

$$
\cot \alpha=\cot \alpha_{0}+\sum_{n=0}^{\infty} c_{n}
$$

It means that the function $\varphi(x, \mu)$ satisfies the boundary condition (1.2) for all $\mu \in \mathbb{C}$.

Find $\beta \in(0, \pi)$ such that $\mu_{n}(q, \alpha, \beta)=\mu_{n}\left(q_{0}, \alpha_{0}, \beta_{0}\right)$ for all $n \geq 0$, i.e., $\varphi(x, \mu)$ should satisfy, at the point $x=\pi$, the boundary condition (1.3)

$$
\varphi\left(\pi, \mu_{n}^{0}\right) \cot \beta+\varphi^{\prime}\left(\pi, \mu_{n}^{0}\right)=0
$$

for this $\beta \in(0, \pi)$. Such $\beta$ (in [6]) is being defined from the following relation

$$
\cot \beta=\cot \beta_{0}+\sum_{n=0}^{\infty} \frac{c_{n} \varphi_{0}^{2}\left(\pi, \mu_{n}^{0}\right)}{1+c_{n} a_{n}^{0}}
$$

Thus Jodeit and Levitan showed that each admissible sequence $\left\{c_{n}\right\}_{n=0}^{\infty}$ generates an isospectral operator $L(q, \alpha, \beta)$, where $q, \alpha$ and $\beta$ are given by formulae (2.3), (2.4) and (2.5), respectively. In this way they obtained all the potentials $q$, with $q^{\prime} \in L^{2}(0, \pi)$, having a given spectrum $\mu_{n}^{0}=\mu_{n}\left(q_{0}, \alpha_{0}, \beta_{0}\right), n \geq 0$.

\section{Proof of Theorem 1.2}

Consider operators $L_{0}=L\left(q_{0}, \alpha_{0}, \beta_{0}\right)$ and $L=L\left(q, \alpha_{0}, \beta\right)$ with the set of norming constants $a_{n}^{0}=a_{n}\left(q_{0}, \alpha_{0}, \beta_{0}\right)$ and $a_{n}=a_{n}\left(q, \alpha_{0}, \beta\right), n \geq 0$, respectively. It is known (see, e.g., [6]) that in this case the kernel $F(x, y)$ of the integral equation (2.2) is

$$
F(x, y)=\sum_{n=0}^{\infty}\left(\frac{1}{a_{n}}-\frac{1}{a_{n}^{0}}\right) \varphi_{0}\left(x, \mu_{n}^{0}\right) \varphi_{0}\left(y, \mu_{n}^{0}\right) .
$$

Since by the condition of Theorem 1.2 the operators $L$ and $L_{0}$ are isospectral, then formulae (2.3)-(2.5) hold. If we compare kernels (2.1) and (3.1), we will refer that $c_{n}=\frac{1}{a_{n}}-\frac{1}{a_{n}^{0}}$. So formulae (2.4) and (2.5) will become

$$
\begin{aligned}
& \cot \alpha=\cot \alpha_{0}+\sum_{n=0}^{\infty}\left(\frac{1}{a_{n}}-\frac{1}{a_{n}^{0}}\right), \\
& \cot \beta=\cot \beta_{0}+\sum_{n=0}^{\infty}\left(a_{n}^{0}-a_{n}\right) \frac{\varphi_{0}^{2}\left(\pi, \mu_{n}^{0}\right)}{\left(a_{n}^{0}\right)^{2}} .
\end{aligned}
$$

Thus, we have all the operators $L(q, \alpha, \beta)$ isospectral with $L\left(q_{0}, \alpha_{0}, \beta_{0}\right)$.

We supposed that $\alpha=\alpha_{0}$, then by formula (3.2) we have

$$
\sum_{n=0}^{\infty}\left(\frac{1}{a_{n}}-\frac{1}{a_{n}^{0}}\right)=0
$$

Since $a_{n} \geq a_{n}^{0}$ for all $n \geq 0$, thus from equation (3.4) it refers that $a_{n}=a_{n}^{0}$ for all $n \geq 0$. Thus, from Marchenko's uniqueness theorem 1.1 we obtain $q(x) \equiv q_{0}(x)$ and $\beta=\beta_{0}$.

This completes the proof. 
Remark 2 From equation (3.4) it follows that the condition $a_{n} \geq a_{n}^{0}$ can be changed with $a_{n} \leq a_{n}^{0}$. From relation (3.3) it follows that we can assume $\beta=\beta_{0}$ instead of $\alpha=\alpha_{0}$ with the condition $a_{n} \geq a_{n}^{0}$ or $a_{n} \leq a_{n}^{0}$, and then we will also obtain $q(x) \equiv q_{0}(x)$ and $\alpha=\alpha_{0}$.

\section{Proof of Theorem 1.4}

Consider an operator $L(q, \alpha, \pi-\alpha)$ and an even operator ${ }^{\mathrm{c}} L(0, \alpha, \pi-\alpha)$.

Levinson proved [20] (see also [21]) that an operator $L$ is even if and only if

$$
\varphi\left(\pi, \mu_{n}\right)=(-1)^{n}, \quad n \geq 0 .
$$

The condition of the theorem means that the operator $L(q, \alpha, \pi-\alpha)$ is isospectral with $L(0, \alpha, \pi-\alpha)$. Since the method of Jodeit and Levitan has described all the isospectral operators for a potential function $q$ with $q^{\prime} \in L^{2}(0, \pi)$, then there exists a sequence $\left\{c_{n}\right\}_{n=0}^{\infty}$ such that $1+c_{n} a_{n}^{0}>0$ for all $n \geq 0,\left\{c_{n}\right\}_{n=0}^{\infty}$ has the properties described in Section 2, and formulae (2.3)-(2.5) hold for operators $L(q, \alpha, \pi-\alpha)$ and $L(0, \alpha, \pi-\alpha)$.

Therefore, taking into account that $q_{0}(x) \equiv 0, \alpha_{0}=\alpha, \beta_{0}=\beta=\pi-\alpha$ and (4.1), relations (2.3)-(2.5), which connect these two operators, will become

$$
\begin{aligned}
& q(x)=2 \frac{d}{d x} K(x, x), \\
& \sum_{n=0}^{\infty} c_{n}=0, \\
& \sum_{n=0}^{\infty} \frac{c_{n}}{1+c_{n} a_{n}^{0}}=0 .
\end{aligned}
$$

If we subtract (4.3) from (4.4), we will obtain

$$
\sum_{n=0}^{\infty} \frac{c_{n}^{2} a_{n}^{0}}{1+c_{n} a_{n}^{0}}=0 .
$$

Since $1+c_{n} a_{n}^{0}>0$ and $a_{n}^{0}>0$ for all $n \geq 0$, then from equation (4.5) we obtain that $c_{n}=$ $0, n \geq 0$. Thus, from equations (2.1), (2.2) and (4.2) it follows that $q(x) \equiv 0$.

Remark 3 We will get the classical Ambarzumyan theorem if we take $\alpha=\pi / 2$.

\section{Appendix: Analogues of Theorem $\mathbf{1 . 2}$}

Consider the $L(q, \alpha, \beta)$ problem. Let $\psi(x, \mu)$ be a solution of equation (1.1), which satisfies the initial conditions

$$
\psi(\pi, \mu)=1, \quad \psi^{\prime}(\pi, \mu)=-\cot \beta .
$$

The eigenvalues $\mu_{n}=\mu_{n}(q, \alpha, \beta), n \geq 0$, are the solutions of the equation

$$
\Phi(\mu):=\varphi(\pi, \mu) \cot \beta+\varphi^{\prime}(\pi, \mu)=0,
$$


or of the equation

$$
\Psi(\mu):=\psi(0, \mu) \cot \alpha+\psi^{\prime}(0, \mu)=0 .
$$

$\Phi(\mu)$ and $\Psi(\mu)$ are called characteristic functions for the operator $L(q, \alpha, \beta)$. In [22] it is proved that characteristic functions and their derivatives are uniquely determined only from their zeros, i.e., from eigenvalues $\left\{\mu_{n}\right\}_{n=0}^{\infty}$. It is easy to see that the functions $\psi\left(x, \mu_{n}\right)$, $n \geq 0$, are the eigenfunctions corresponding to the eigenvalue $\mu_{n}$. The squares of the $L^{2}$ norm of these eigenfunctions

$$
b_{n}=b_{n}(q, \alpha, \beta):=\int_{0}^{\pi}\left|\psi\left(x, \mu_{n}\right)\right|^{2} d x, \quad n \geq 0,
$$

are called norming constants.

Since all the eigenvalues of $L(q, \alpha, \beta)$ are simple, then there exist constants $\kappa_{n}=$ $\kappa_{n}(q, \alpha, \beta), n \geq 0$, such that

$$
\varphi\left(x, \mu_{n}\right)=\kappa_{n} \psi\left(x, \mu_{n}\right)
$$

The theorem of uniqueness of Harutyunyan (see [21]) states the following.

\section{Theorem A.1 If}

$$
\begin{aligned}
& \mu_{n}(q, \alpha, \beta)=\mu_{n}\left(q_{0}, \alpha_{0}, \beta_{0}\right), \\
& \kappa_{n}(q, \alpha, \beta)=\kappa_{n}\left(q_{0}, \alpha_{0}, \beta_{0}\right),
\end{aligned}
$$

for all $n \geq 0$, then $\alpha=\alpha_{0}, \beta=\beta_{0}$ and $q(x)=q_{0}(x)$ almost everywhere.

From (1.4), (A.1) and (A.2) it follows

$$
\kappa_{n}=\varphi\left(\pi, \mu_{n}\right)=\psi^{-1}\left(0, \mu_{n}\right) .
$$

There is a relationship between norming constants and characteristic functions (see, e.g., $[6,21])$

$$
\begin{aligned}
& a_{n}=\left|\varphi\left(\pi, \mu_{n}\right)\right|\left|\dot{\Phi}\left(\mu_{n}\right)\right|, \\
& b_{n}=\left|\psi\left(0, \mu_{n}\right)\right|\left|\dot{\Psi}\left(\mu_{n}\right)\right|,
\end{aligned}
$$

where the dot over $\Phi$ (or over $\Psi$ ) denotes the derivative of $\Phi(\mu)$ with respect to $\mu$. From equations (A.3) and (A.4) we obtain

$$
a_{n}=\left|\kappa_{n}\right|\left|\dot{\Phi}\left(\mu_{n}\right)\right| .
$$

Consider two isospectral operators $L(q, \alpha, \beta)$ and $L\left(q_{0}, \alpha_{0}, \beta_{0}\right)$. The following formulae, analogues to (2.4) and (2.5), can be obtained for $\kappa_{n}$ :

$$
\cot \alpha=\cot \alpha_{0}+\sum_{n=0}^{\infty} \frac{1}{\left|\dot{\Phi}\left(\mu_{n}^{0}\right)\right|}\left(\frac{1}{\left|\kappa_{n}\right|}-\frac{1}{\left|\kappa_{n}^{0}\right|}\right),
$$




$$
\cot \beta=\cot \beta_{0}+\sum_{n=0}^{\infty} \frac{\left|\kappa_{n}^{0}\right|-\left|\kappa_{n}\right|}{\left|\dot{\Phi}\left(\mu_{n}^{0}\right)\right|} .
$$

From Theorem A.1 and formulae (2.3), (A.7), (A.8), a new statement, similar to Theorem 1.2, can be proven for $\kappa_{n}$ as follows.

Theorem A.2 Let $q^{\prime} \in L_{\mathbb{R}}^{2}(0, \pi)$. If

$$
\begin{aligned}
& \mu_{n}\left(q, \alpha_{0}, \beta\right)=\mu_{n}\left(q_{0}, \alpha_{0}, \beta_{0}\right), \\
& \left|\kappa_{n}\left(q, \alpha_{0}, \beta\right)\right| \geq\left|\kappa_{n}\left(q_{0}, \alpha_{0}, \beta_{0}\right)\right|,
\end{aligned}
$$

for all $n \geq 0$, then $\beta=\beta_{0}$ and $q(x) \equiv q_{0}(x)$.

Remark 4 Instead of $\alpha=\alpha_{0}$, we can fix $\beta=\beta_{0}$ and/or replace the inequality sign (' $\geq$ ') with less than or equal sign (' $\leq$ '). Even so, the result is valid. Similar theorems can be proven for $\varphi\left(\pi, \mu_{n}\right)$.

Remark 5 Since the uniqueness theorem of Marchenko is also true for norming constants $b_{n}$, taking into consideration relations (A.2), (A.3) and (A.5), analogues to Theorem 1.2 can be proven for $\psi\left(0, \mu_{n}\right)$ and $b_{n}$.

\section{Acknowledgements}

The author would like to thank the referees for their helpful comments and suggestions. The author is also grateful to professor TN Harutyunyan for valuable remarks and discussions.

\section{Funding}

This work was supported by the RA MES State Committee of Science, in the frames of the research project No.15T-1A392.

\section{Competing interests}

The author declares that he has no competing interests.

\section{Author's contributions}

The author read and approved the final manuscript.

\section{Endnotes}

a The theorem of Marchenko is more general, see e.g. [5, 23-25].

b Here $F(x, y)$ is a kernel of integral equation (2.2), where $x$ is a parameter, $F(x, y)$ is a known function and $K(x, y)$ is an unknown function, as functions of $y$.

c A problem $L(q, \alpha, \beta)$ is said to be even if $q(x)=q(\pi-x)$ and $\alpha+\beta=\pi$.

\section{Publisher's Note}

Springer Nature remains neutral with regard to jurisdictional claims in published maps and institutional affiliations.

Received: 25 February 2017 Accepted: 18 May 2017 Published online: 30 May 2017

\section{References}

1. Naimark, MA: Linear Differential Operators. Nauka, Moscow (1969) (in Russian)

2. Marchenko, VA: Sturm-Liouville Operators and Its Applications. Naukova Dumka, Kiiv (1977) (in Russian)

3. Levitan, BM, Sargsyan, IS: Sturm-Liouville and Dirac Operators. Nauka, Moskva (1988) (in Russian)

4. Yurko, VA: An Introductions to the Theory of Inverse Spectral Problems. Fizmatlit, Moskva (2007) (in Russian)

5. Marchenko, VA: Concerning the theory of a differential operator of the second order. Dokl. Akad. Nauk SSSR 72, 457-460 (1950) (in Russian)

6. Jodeit, M, Levitan, BM: The isospectrality problem for the classical Sturm-Liouville equation. Adv. Differ. Equ. 2(2), 297-318 (1997)

7. Ambarzumyan, VA: Über eine frage der eigenwertsththeori. Z. Phys. 53, 690-695 (1929)

8. Kuznezov, NV: Extensions of VA Ambarzumyan theorem. Dokl. Akad. Nauk 146, 1259-1262 (1962)

9. Chakravarty, NK, Acharyya, SK: On an extension of the theorem of VA Ambarzumyan. Proc. R. Soc. Edinb. A 110, 79-84 (1988) 
10. Chern, H-H, Law, CK, Wang, H-J: Extension of Ambarzumyan's theorem to general boundary conditions. J. Math. Anal. Appl. 263, 333-342 (2001)

11. Chern, H-H, Law, CK, Wang, H-J: Corrigendum to "Extension of Ambarzumyan's theorem to general boundary conditions". J. Math. Anal. Appl. 309, 764-768 (2005)

12. Yang, C-F, Huang, Z-Y, Yang, X-P: Ambarzumyan's theorems for vectorial Sturm-Liouville systems with coupled boundary conditions. Taiwan. J. Math. 14(4), 1429-1437 (2010)

13. Yang, Y, Wang, F: New Ambarzumyan's theorems for differential operators with operator coefficient. Adv. Math. 40(6), 749-755 (2011)

14. Yurko, VA: On Ambarzumyan-type theorems. Appl. Math. Lett. 20, 506-509 (2013)

15. Yilmaz, E, Koyunbakan, H: Ambarzumyan type theorem for a matrix valued quadratic Sturm-Liouville problem. Comput. Model. Eng. Sci. 99(6), 463-471 (2014)

16. Isaacson, EL, Trubowitz, E: The inverse Sturm-Liouville problem, I. Commun. Pure Appl. Math. 36, 767-783 (1983)

17. Isaacson, EL, McKean, HP, Trubowitz, E: The inverse Sturm-Liouville problem, II. Commun. Pure Appl. Math. 37, 1-11 (1984)

18. Dahlberg, BEJ, Trubowitz, E: The inverse Sturm-Liouville problem, III. Commun. Pure Appl. Math. 37, 255-267 (1984)

19. Poshel, J, Trubowitz, E: Inverse Spectral Theory. Academic Press, New York (1987)

20. Levinson, N: The inverse Sturm-Liouville problem. Mat. Tidsskr., B 1949, 25-30 (1949)

21. Harutyunyan, TN: On a uniqueness theorem in the inverse Sturm-Liouville problem. Mat. Vesn. 61, 139-147 (2009)

22. Harutyunyan, TN: Representation of the norming constants by two spectra. Electron. J. Differ. Equ. 2010, 159 (2010)

23. Marchenko, VA: Concerning the theory of a differential operator of the second order. Tr. Mosk. Mat. Obŝ. 1, 327-420 (1952) (in Russian)

24. Levitan, BM: Generalized Translation Operators and Some of Their Applications. Fizmatgiz, Moskva (1962) (in Russian)

25. Freiling, G, Yurko, VA: Inverse Sturm-Liouville Problems and Their Applications. Nova Science Publishers, New York (2001)

\section{Submit your manuscript to a SpringerOpen ${ }^{\circ}$ journal and benefit from:}

- Convenient online submission

Rigorous peer review

- Immediate publication on acceptance

- Open access: articles freely available online

- High visibility within the field

- Retaining the copyright to your article 